\title{
Composite pairs and effective two-body scattering in a many-body medium
}

DOI:

10.1103/PhysRevA.13.1570

\section{Document Version}

Final published version

Link to publication record in Manchester Research Explorer

\section{Citation for published version (APA):}

Bishop, R. F., Ghassib, H. B., \& Strayer, M. R. (1976). Composite pairs and effective two-body scattering in a many-body medium. Physical Review A (Atomic, Molecular and Optical Physics), 13, 1570-1580.

https://doi.org/10.1103/PhysRevA.13.1570

\section{Published in:}

Physical Review A (Atomic, Molecular and Optical Physics)

\section{Citing this paper}

Please note that where the full-text provided on Manchester Research Explorer is the Author Accepted Manuscript or Proof version this may differ from the final Published version. If citing, it is advised that you check and use the publisher's definitive version.

\section{General rights}

Copyright and moral rights for the publications made accessible in the Research Explorer are retained by the authors and/or other copyright owners and it is a condition of accessing publications that users recognise and abide by the legal requirements associated with these rights.

\section{Takedown policy}

If you believe that this document breaches copyright please refer to the University of Manchester's Takedown Procedures [http://man.ac.uk/04Y6Bo] or contact uml.scholarlycommunications@manchester.ac.uk providing relevant details, so we can investigate your claim.

\section{OPEN ACCESS}




\title{
Composite pairs and effective two-body scattering in a many-body medium
}

\author{
R. F. Bishop \\ Department of Theoretical Physics, Manchester University, Manchester M13 9PL, England \\ and Science Research Council, Daresbury Laboratory, Daresbury, Warrington WA4 4AD, England \\ H. B. Ghassib \\ Department of Theoretical Physics, Manchester University, Manchester, England \\ and Department of Physics, University of Jordan, Amman, Jordan* \\ M. R. Strayer \\ Science Research Council, Daresbury Laboratory, Daresbury, England
}

(Received 20 October 1975)

\begin{abstract}
The generalized off-shell scattering of pairs of constituent fermions embedded in an infinite system of identical particles having thermodynamic properties characterized by arbitrary values of the temperature and chemical potential, is examined using a temperature-dependent Green's-function formalism, which treats particleparticle and hole-hole excitations symmetrically. The important features which emerge are (i) the possible existence of bound-state pairs (of quasiparticles which are linear combinations of particle and hole states) which correspond to poles in the scattering amplitude at negative total energies depending parametrically on the properties of the medium and the total momentum of the interacting pair; (ii) a generalized unitarity condition from which one obtains a parametrization of the on-shell positive-energy scattering amplitude by a set of real effective phase shifts; (iii) that these phase shifts completely determine the large-separation asymptotic behavior of the scattering (positive energy) pair wave functions in a manner which is form equivalent to two-particle scattering in free space; and (iv) that the small- and large-momentum asymptotic limits of the phase shifts are related to the number of bound-state pairs in the medium via an analogous, but modified, form of Levinson's theorem for free scattering. The effects of these pairing singularities on the singleparticle excitation spectrum, and hence on the thermodynamic behavior of the system are outlined.
\end{abstract}

\section{INTRODUCTION}

Historically, the scattering of constituent pairs of particles within an infinite background or medium of like particles, has played a central role in understanding the observed behavior of manyfermion systems. ${ }^{1}$ While not directly physically measurable, an effective scattering amplitude serves as a useful precursor in relating thermodynamic and average single-particle behavior in phenomenological theories; ${ }^{2}$ and as the lowestorder building block in an often essential rearrangement of the perturbation series in microscopic perturbation theories. ${ }^{3}$ Perhaps the most successful application of these concepts resulted from an analysis of the scattering of electron pairs in a degenerate electron gas ${ }^{4}$ (the Cooper problem), for which it was demonstrated that the formation of bound electron pairs in the medium gives rise to the phenomenon of superconductivity.

While it is generally true that the existence of bound-state pairs in a many-body medium can be linked to singularities in the effective scattering amplitude, this alone does not suffice to guarantee that the pairs will possess significant boson-like properties. In this context, the problem of condensation of composite bosons has been investigated by Yang, ${ }^{5}$ and by Kohn and Sherrington, ${ }^{6}$ who show that condensates of these composite particles will not exhibit superfluid properties unless they also possess a long-range (macroscopic) off-diagonal order. It is also believed that, in fermion systems governed by strongly repulsive short-range forces, bound-pair formation is effectively hindered by the repulsive core. This belief followed from a series of computations on the ${ }^{3} \mathrm{He}$ system initiated by Brueckner and Gammel, ${ }^{8}$ in which it was found that the effective $T$ matrix (the off-shell generalization of the effective scattering amplitude) was strongly repulsive in the $S$-wave channel, and which led to the conjecture that liquid ${ }^{3} \mathrm{He}$ is an anisotropic superfluid in the ground state. In spite of the validity of this conjecture, the reasoning leading to it is not precisely correct in that it has been explicitly demonstrated that bound-pair formation can occur in the ${ }^{3} \mathrm{He} S$-wave interaction. ${ }^{9}$ As was emphasized in Ref. 9, these bound-state singularities arise quite naturally when attempting to describe the normal ${ }^{3} \mathrm{He}$ liquid within the timedependent Green's function theory of Galitskii and Migdal, ${ }^{10}$ and are presumably unrelated to the anistropic superfluid phases.

Using techniques of quantum-field theory, a formalism has been developed which is suitable for analyzing zero-temperature many-fermion 
systems, using very general properties of the bare interaction without specifying the nature of the fermions; and hence a theoretical framework is available which is valid for both nuclear matter and liquid ${ }^{3} \mathrm{He}$. The theory also has a completely "natural" generalization to systems at finite temperatures, ${ }^{11}$ in the sense that all of the diagrams enumerated at finite temperature can be placed in a one-to-one correspondence with those at zero temperature. The only basic difference arises from the fact that the one-body propagator becomes explicitly temperature dependent.

Despite the appealing theoretical arguments ${ }^{12}$ in favor of the Green's function method, practical ly all of the computations to date ${ }^{13}$ have been carried out within the framework of BruecknerBethe-Goldstone (BG) theory. ${ }^{14}$ Our underlying philosophy is that each theory is formally exact when carried to an infinite order, and hence must yield identical results in this limit. However, when terminated at some finite order, they are manifestly different theories and are to be expected to give different results. In particular, the so-called ladder diagrams defining the effective two-body $T$ matrices in each formalism are by no means the same. They subsume quite different physics in each case and possess quite different properties.

Nuclear matter and liquid ${ }^{3} \mathrm{He}$ are both fermion systems in which the bare two-particle interaction becomes strongly repulsive at small interparticle separations, For this reason it is neces sary to (exactly) sum the ladder diagrams (and thereby obtain an off-shell transition amplitude or $T$ matrix), and then to rearrange the basic many-body perturbation series so that the bare potential never explicitly appears. In the Galitskii formalism, the sum of the non-time-ordered Feynman ladder diagrams defines an effective two-body $T$ matrix, namely the Galitskii-Feynman (GF) $T$ matrix. These diagrams, obtained by allowing an arbitrary number of successive twobody bare potential interactions in which the intermediate scattering states are either pairs of particles outside or holes inside the Fermi sea, can be formally summed by an integral equation, the solution to which is the GF T matrix. Conversely, the corresponding BG $T$ matrix allows only those time orderings which correspond to pairs of particles outside the Fermi sea. Holehole scattering is not included at all in the BG $T$ matrix, whereas it is explicitly included on an equal and symmetric footing to particle-particle scattering in the GF case. The difference is important in both nuclear matter and ${ }^{3} \mathrm{He}$ systems in so far as it has been demonstrated ${ }^{9}$ that in each case the GF $T$ matrix has singularities (first- order poles) which are absent in the corresponding BG $T$ matrix, and which correspond to the formation of bound-state pairs in the many-body medium. Calculations were performed in Ref. 9 specifically for the ${ }^{3} \mathrm{He}$ and nuclear matter systems using typical phenomenological $\mathrm{He}-\mathrm{He}$ and nucleon-nucleon potentials, but many of the main conclusions are almost certainly true for manyfermion systems in general. The main results of Ref. 9 can be summarized as follows: (i) The singularity occurs as a first-order pole in the energy variable of the $S$-wave GF $T$ matrix, having a residue which factorizes in terms of the incoming and outgoing relative momentum variables. (ii) The position of the pole (i.e., the binding energy of the pair, or alternatively the spectrum of the composite boson) is a function of two independent thermodynamic variables characterizing the medium (i.e., density and temperature, or chemical potential and temperature), and also of the total linear momentum of the interacting pair. (iii) There exist critical values of the density or chemical potential below which, and of the temperature and total momentum above which, the pole vanishes. (iv) Whenever hole-hole scattering is excluded from the intermediate states, as in the BG $T$ matrix, the pole appears to be completely absent.

The effects of these singularities are difficult to assess quantitatively, primarily because complete computations using the Green's-function theory are difficult-appreciably more difficult than the corresponding BG calculations-but in general can be understood in the following way. The pole occurs in the GF $T$ matrix from which the "exact" proper self-energy function can be obtained to all orders of perturbation theory. The lowest-order contribution to the proper selfenergy involves only the first power of the GF $T$ matrix, and requires an integration over the center-of-mass (CM) energy variable. The effect of the integration is thus to "soften" the singularity. In higher orders each $T$ matrix is accompanied by a corresponding integration, and thus the "exact" single-particle spectrum should not exhibit such marked singular behavior as the $T$ matrix itself. The pole will, however, manifest itself in the building up of a single-particle spectrum having a particular analytic structure due to it.

The purpose of this study is simultaneously to examine the scattering properties of pairs of constituent particles embedded in a many-fermion background, focusing on those features of the system related to the formation of bound-state singularities (composite bosons), and to establish a foundation for subsequent finite-temperature 
calculations for the proper self-energy function, in which the bound-state poles are expected to play a central role. The basic philosophy and motivation are that the formation of pairing singularities in a fermion system is both of interest in its own right, and necessary to fully under stand before attempting approximate calculations of the average single-particle properties of the system. In particular, the existence of boundstate poles place important constraints on the fully off-shell temperature-dependent GF $T$ matrix. Also, using arguments based on timereversal invariance and hermiticity, a generalized two-particle unitarity relation is obtained. This requires that the on-shell (positive energy) GF $T$ matrix in each relative partial wave channel is, apart from real kinematic and phase-space factors, unimodular, and hence can be parametrized in terms of a real phase shift. This is an implicit function of the total linear momentum of the interacting pair, and of two independent thermodynamic variables (e.g., temperature and chemical potential) characterizing the medium. In a complementary fashion, nonlocal wave equations are derived for the temperature-dependent pair wave functions. The asymptotic form of these wave functions for positive-energy solutions is found, assuming outgoing-wave boundary conditions at infinity, in terms of the on-shell parametrization of the GF T matrix. Using these wave functions and an assumed completeness relation, the number of bound-state pairs in the medium is related to the asymptotic behavior of the effective phase shifts via a modified form of Levinson's theorem. A quantitative correspondence between two-particle scattering in free space and the scattering of pairs in a manyfermion medium is thereby established.

Throughout the remainder of this work we shall restrict our discussion to a system of fermions interacting via a finite-range, square-integrable, two-body central potential, having the basic feature of being everywhere attractive except at short interparticle distances where it becomes strongly repulsive. It is also assumed, for ease of discussion, that the free two-particle system does not have any bound-states, although this condition can readily be relaxed. The real-time temperature-dependent formalism is outlined in Sec. II, and the basic properties of the finitetemperature GF $T$ matrix are developed along with the dispersion relation for the bound-state poles, and the generalized unitarity relation. The nonlocal wave equations and their asymptotic solutions are given in Sec. III, where an appropriate generalization of Levinson's theorem is proved. The important features of this calculation are summarized, and possible consequences on the singularity structure for the proper self-energy are drawn in Sec. IV.

\section{REAL-TIME TEMPERATURE-DEPENDENT FORMALISM}

The basic principles delineating the application of Feynman-diagram techniques to many-body systems have been set forth by Galitskii and Migdal $^{10}$ at zero temperature, and have been extended to finite temperature by Abrikosov et al. ${ }^{11}$ and many others. Compact descriptions of the temperature formalism are extensively available in the literature ${ }^{15}$ and we shall accordingly sidestep any detailed discussion of the formal theory. The finite-temperature theory differs substantially from the zero-temperature theory. In brief, one assumes that the equilibrium thermodynamic properties and average single-particle excitations of the system can be completely specified by a knowledge of the real-time single-particle Green's function, which in turn can be uniquely determined either from the imaginary-time one-particle propagator or by direct calculation, using the first Dyson equation, ${ }^{16}$

$$
\bar{G}^{-1}(p)=\bar{G}_{0}^{-1}(p)+\bar{\Sigma}^{*}(p),
$$

where $p$ represents a four-momentum variable, $p$ $=\left(\overrightarrow{\mathrm{p}}, p_{0}\right)$. The identification of the various terms in Eq. (1) is as follows. The single-particle propagator in the noninteracting system is $\bar{G}_{0} ; \bar{\Sigma}^{*}$ is the (exact) proper self-energy function which must be determined in perturbation theory using the Feynman rules; and $\bar{G}$ is the temperature Green's function which specifies the propagation of a single particle or hole in the fully interacting system. The bar over each quantity in Eq. (1) is used here and henceforth to denote the implicit dependence on the two thermodynamic variables, temperature $\beta^{-1}$ and chemical potential $\mu$. The behavior of the system in thermal equilibrium is thus known by calculating observables in terms of the volume $V$ and the Feynman single-particle propagator $\bar{G}$. For example, the (fixed) total number of particles in the system is given by

$$
N\left(\beta^{-1}, V, \mu\right)=\lim _{\eta \rightarrow 0^{+}} g V \int \frac{d^{4} p}{(2 \pi)^{4}} e^{i p_{0} \eta} \bar{G}(p),
$$

where $g$ is the degeneracy factor corresponding to the internal quantum numbers such as spin and isospin (e.g., $g=2$ for spin- $-\frac{1}{2}$ fermions of one type). Using this equation, the dependence of other quantities on the chemical potential can be eliminated in favor of the number of particles in the system, or equivalently the density, thus maintaining a close correspondence with the zero- 


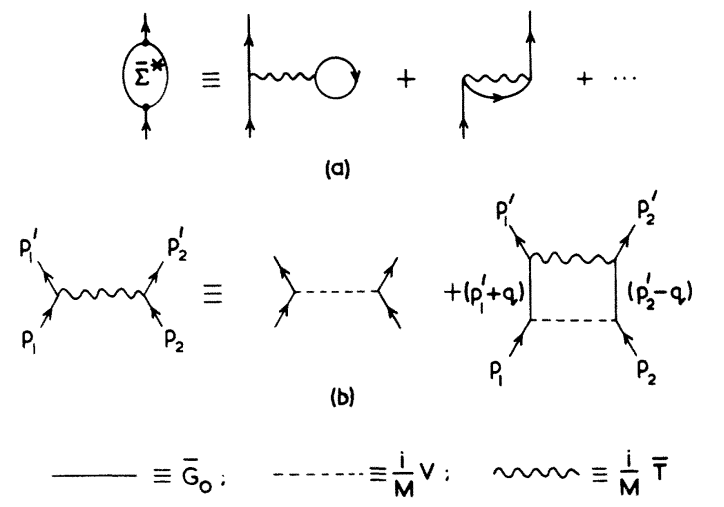

FIG. 1. (a) Lowest-order $T$ matrix contributions to the proper self-energy. (b) Ladder diagrams summed pictorially to give the $T$-matrix equation.

temperature theory.

At finite temperatures, the complex imaginary single-particle spectrum corresponds to the excitations of particles moving in an external po- tential $\mu$, and having an internal energy given by the poles of the exact one-body propagator $\bar{G}$,

$$
\bar{\epsilon}(\overrightarrow{\mathrm{p}})+i \bar{\gamma}(\overrightarrow{\mathrm{p}})=\bar{p}_{0}+\mu,
$$

where $\bar{p}_{0}=\bar{p}_{0}(\overrightarrow{\mathrm{p}})$ is the solution to the equation

$$
\bar{p}_{0}=\left(\overrightarrow{\mathrm{p}}^{2}-\mu\right)+\bar{\Sigma} *\left(\overrightarrow{\mathrm{p}}, \bar{p}_{0}\right) .
$$

The functions $\bar{\epsilon}$ and $\bar{\gamma}$ are both real, and depend implicitly on the temperature and chemical potential of the system. It should be noted that throughout this work we use units such that $2 M$ $=\hbar=k_{B}=1$ ( $M$ being the particle mass, and $k_{B}$ Boltzmann's constant). As in the case of the thermodynamic functions, the dependence of the single-particle excitation spectrum on the chemical potential can be eliminated in favor of the number density of the particles using Eq. (2).

The lowest-order GF $T$-matrix contributions to $\bar{\Sigma}^{*}$ are shown in Fig. 1(a), and the ladder sum of non-time-ordered diagrams defining the $T$ matrix is shown in Fig. 1(b). Neglecting any possible spin-dependence, the GF $T$ matrix as given by Eq. (4)

$$
\begin{aligned}
2 i \bar{T}\left(p_{1}, p_{2} ; p_{1}^{\prime}, p_{2}^{\prime}\right)= & i V\left(p_{1}, p_{2} ; p_{1}^{\prime}, p_{2}^{\prime}\right) \\
& +2 i^{2}(2 \pi)^{-4} \int d^{4} q V\left(p_{1}, p_{2} ; p_{1}+q, p_{2}-q\right) \bar{G}_{0}\left(p_{1}+q\right) \bar{G}_{0}\left(p_{2}-q\right) \bar{T}\left(p_{1}+q, p_{2}-q ; p_{1}^{\prime}, p_{2}^{\prime}\right)
\end{aligned}
$$

formally depends on 16 initial - and final-state momentum variables. As in Eq. (1), $\bar{G}_{0}$ is the noninteracting temperature Green's function,

$$
\begin{aligned}
& \bar{G}_{0}(p)=\frac{n_{0}(\overrightarrow{\mathrm{p}})}{p_{0}-\left(\overrightarrow{\mathrm{p}}^{2}-\mu\right)+i \eta}+\frac{1-n_{0}(\overrightarrow{\mathrm{p}})}{p_{0}-\left(\overrightarrow{\mathrm{p}}^{2}-\mu\right)-i \eta}, \\
& n_{0}(x)=\left(1+e^{-\beta\left(x^{2}-\mu\right)}\right)^{-1} .
\end{aligned}
$$

The quantity $\eta$ is a positive infinitesimal, and $n_{0}(x)$ is the usual Fermi distribution function. Conservation of total four-momentum in Eq. (4) reduces the number of linearly independent four- momenta to three. The potential $V$ is also a static central two-body interaction which depends only on the magnitude of the three-momentum transfer, $V=V\left(\left|\overrightarrow{\mathrm{p}}-\overrightarrow{\mathrm{p}}^{\prime}\right|\right)$; where relative and $\mathrm{CM}$ momentum variables are defined as

$$
\begin{aligned}
& p=\frac{1}{2}\left(p_{1}-p_{2}\right), \quad p^{\prime}=\frac{1}{2}\left(p_{1}^{\prime}-p_{2}^{\prime}\right), \\
& P=\frac{1}{2}\left(p_{1}+p_{2}\right)=\frac{1}{2}\left(p_{1}^{\prime}+p_{2}^{\prime}\right) .
\end{aligned}
$$

Using the above representation and employing the substitution $q \rightarrow q-p, \mathrm{Eq}$. (4) can be rewritten in the form

$\bar{T}\left(P+p, P-p ; P+p^{\prime}, P-P^{\prime}\right)=u\left(\left|\overrightarrow{\mathrm{p}}-\overrightarrow{\mathrm{p}}^{\prime}\right|\right)+2 i(2 \pi)^{-4} \int d^{4} q u(|\overrightarrow{\mathrm{p}}-\overrightarrow{\mathrm{q}}|) \bar{G}_{0}(P+q) \bar{G}_{0}(P-q) \bar{T}\left(P+q, P-q ; P+p^{\prime}, P-p^{\prime}\right)$, where $u \equiv M \hbar^{-2} V=\frac{1}{2} V$. In this form one can easily see that the kernel and inhomogeneous term are independent of the variables $p_{0}$ and $p_{0}^{\prime}$, and hence that the $T$ matrix is only a function of ten linearly independent energy-momentum variables, which we write as $\bar{T}\left(\overrightarrow{\mathrm{p}}, \overrightarrow{\mathrm{p}}^{\prime} ; P_{0}, \overrightarrow{\mathrm{P}}\right)$, and which satisfies the integral equation

$$
\bar{T}\left(\overrightarrow{\mathrm{p}}, \overrightarrow{\mathrm{p}}^{\prime} ; \boldsymbol{P}_{0}, \overrightarrow{\mathrm{P}}\right)=u\left(\left|\overrightarrow{\mathrm{p}}-\overrightarrow{\mathrm{p}}^{\prime}\right|\right)-(2 \pi)^{-3} \int d \overrightarrow{\mathrm{q}} u(|\overrightarrow{\mathrm{p}}-\overrightarrow{\mathrm{q}}|)\left[\frac{2}{i} \int_{-\infty}^{\infty} \frac{d q_{0}}{2 \pi} \bar{G}_{0}(P+q) \bar{G}_{0}(P-q)\right] \bar{T}\left(\overrightarrow{\mathrm{q}}, \overrightarrow{\mathrm{p}}^{\prime} ; P_{0}, \overrightarrow{\mathrm{P}}\right) .
$$

The integration over $q_{0}$ can now be easily performed, and the resulting quantity in brackets in the above equation is seen to be a function of the variables $\overrightarrow{\mathrm{q}}, \overrightarrow{\mathrm{P}}$, and $s$, where the CM energy variable $s$ is defined 
as $s \equiv P_{0}+\mu-\overrightarrow{\mathrm{P}}^{2}$. The $T$-matrix equation (4) now takes the form

$$
\begin{aligned}
& \bar{T}\left(\overrightarrow{\mathrm{p}}, \overrightarrow{\mathrm{p}}^{\prime} ; s, \overrightarrow{\mathrm{P}}\right)=u\left(\left|\overrightarrow{\mathrm{p}}-\overrightarrow{\mathrm{p}}^{\prime}\right|\right)-(2 \pi)^{-3} \int d \overrightarrow{\mathrm{q}} u(|\overrightarrow{\mathrm{p}}-\overrightarrow{\mathrm{q}}|)\left[g_{0}(\overrightarrow{\mathrm{q}}, s) Q(\overrightarrow{\mathrm{q}}, \overrightarrow{\mathrm{p}})-g_{0}^{*}(\overrightarrow{\mathrm{q}}, s) \tilde{\mathrm{Q}}(\overrightarrow{\mathrm{q}}, \overrightarrow{\mathrm{P}})\right] T\left(\overrightarrow{\mathrm{q}}, \overrightarrow{\mathrm{p}}^{\prime} ; s, \overrightarrow{\mathrm{P}}\right) ; \\
& g_{0}(\overrightarrow{\mathrm{q}}, s)=\left(\overrightarrow{\mathrm{q}}^{2}-s-i \eta\right)^{-1}, \quad Q(\overrightarrow{\mathrm{q}}, \overrightarrow{\mathrm{P}})=n_{0}(\overrightarrow{\mathrm{P}}+\overrightarrow{\mathrm{q}}) n_{0}(\overrightarrow{\mathrm{P}}-\overrightarrow{\mathrm{q}}), \quad \tilde{Q}(\overrightarrow{\mathrm{q}}, \overrightarrow{\mathrm{P}})=\left[1-n_{0}(\overrightarrow{\mathrm{P}}+\overrightarrow{\mathrm{q}})\right]\left[1-n_{0}(\overrightarrow{\mathrm{P}}-\overrightarrow{\mathrm{q}})\right] .
\end{aligned}
$$

The $T$ matrix is thus clearly seen to represent the scattering of pairs of particles or pairs of holes, from an initial relative momentum state $\vec{p}$ to a final momentum state $\vec{p}^{\prime}$, in the CM frame of the pair having a total momentum $2 \overrightarrow{\mathrm{P}}$ and energy $2 s$.

Equation (6) is usually analyzed further by making use of the assumption that the Fermi distribution functions are not too sensitive to the orientation of the total momentum vector of the pair. In this approximation the GF $T$ matrix can be expanded as an uncoupled partial-wave series

$$
\bar{T}_{l}\left(p, p^{\prime} ; s, P\right)=u_{l}\left(p, p^{\prime}\right)-\left(2 \pi^{2}\right)^{-1} \int_{0}^{\infty} d q q^{2} u_{l}(p, q)\left[Q(q, P) g_{0}(q, s)-\tilde{Q}(q, P) g_{0}^{*}(q, s)\right] \bar{T}_{l}\left(q, p^{\prime} ; s, P\right),
$$

where

$$
\begin{aligned}
& \bar{T}\left(\overrightarrow{\mathrm{p}}, \overrightarrow{\mathrm{p}}^{\prime} ; s, P\right)=\sum_{l}(2 l+1) \bar{T}_{l}\left(p, p^{\prime} ; s, P\right) P_{l}\left(\hat{p} \cdot \hat{p}^{\prime}\right), \\
& u\left(\left|\overrightarrow{\mathrm{p}}-\overrightarrow{\mathrm{p}}^{\prime}\right|\right)=\sum_{l}(2 l+1) u_{l}\left(p, p^{\prime}\right) P_{l}\left(\hat{p} \cdot \hat{p}^{\prime}\right) .
\end{aligned}
$$

In this context the angle-averaging reduction of the functions $Q$ and $\tilde{Q}$ can be performed analytically with the result

$$
\begin{aligned}
Q(q, P) & \equiv \frac{1}{2} \int_{-1}^{1} d x Q(\overrightarrow{\mathrm{q}}, \overrightarrow{\mathrm{P}}) \\
& =\left[2 \beta q P\left\{1-\exp \left[-2 \beta\left(P^{2}+q^{2}-\mu\right)\right]\right\}\right]^{-1} L(q, P), \\
\tilde{Q}(q, P) & \equiv \frac{1}{2} \int_{-1}^{1} d x \tilde{Q}(\overrightarrow{\mathrm{q}}, \overrightarrow{\mathrm{P}}) \\
& =\left[2 \beta q P\left\{\exp \left[2 \beta\left(P^{2}+q^{2}-\mu\right)\right]-1\right\}\right]^{-1} L(q, P), \\
L(q, P) & =\ln \left(\frac{\cosh \left\{\frac{1}{2} \beta\left[(P+q)^{2}-\mu\right]\right\}}{\cosh \left\{\frac{1}{2} 3\left[(P-q)^{2}-\mu\right]\right\}}\right),
\end{aligned}
$$

where $x \equiv \hat{q} \cdot \hat{P}$.

Equation (7) has been studied numerically for a series of phenomenological helium interatomic potentials appropriate to the liquid ${ }^{3} \mathrm{He}$ system, and it has been reported ${ }^{9,17}$ that the $S$-wave $(l=0)$ GF $T$ matrix possesses a first-order pole at negative energies $s=\bar{s}_{l}(P)<0$, of the form

$\lim _{s \rightarrow \bar{s}_{l}(P)}\left[s-\bar{s}_{l}(P) \mid \bar{T}_{l}\left(p, p^{\prime} ; s, P\right)=-\bar{R}_{l}(p, P) \bar{R}_{l}\left(p^{\prime}, P\right)\right.$,

where again the bar over the various quantities denotes the implicit dependence on the parameters $\beta$ and $\mu$. The residue functions $\bar{R}_{l}$ and the dispersion relation for the pair spectrum in the $l$ th relative partial wave can easily be found by substituting Eq. (9) into Eq. (7) to yield

$$
\begin{aligned}
\bar{R}_{l}(p, P)=-\int_{0}^{\infty} & \frac{d q q^{2}}{2 \pi^{2}} u_{l}(p, q) \\
& \times\left(\frac{Q(q, P)-\tilde{Q}(q, P)}{q^{2}-\bar{s}_{l}(P)}\right) \bar{R}_{l}(q, P) .
\end{aligned}
$$

Working by analogy with free two-particle scattering (in vacuo), the interpretation of the pole as given in Ref. 9 is that it corresponds to the formation of bound states in the medium which are linear combinations of pairs of particles and pairs of holes, and which obey a dispersion relation $\bar{\epsilon}(P)=2 \bar{s}_{0}(P)$. The function $\bar{\epsilon}(P)$, as computed in Ref. 17 using the modified Frost-Musulin potential $1^{18,19}$ appropriate to liquid ${ }^{3} \mathrm{He}$, is dis played in Fig. 2 for fixed values of 3 and $\mu$. For this interaction, $\bar{\epsilon}$ can be parametrized to a very high degree of accuracy by the functional form

$$
\bar{\epsilon}(P)=\bar{\epsilon}_{0}\left|1-\left(P / \bar{P}_{c}\right)^{2}\right|^{\bar{\lambda}} .
$$

The bound pair exists only for $P<\bar{P}_{c}$, the critical total momentum; and each of the parameters $\bar{\epsilon}_{0}, \bar{P}_{c}$, and $\bar{\lambda}$ is an implicit function of $\beta$ and $\mu$.

In what follows, it will prove useful to evolve an operator notation, free from any particular representation, in which the GF $T$-matrix equation can be written as

$\bar{T}(s, P)=u-u\left[Q(P) g_{0}(s)-\tilde{Q}(P) g_{0}^{\dagger}(s)\right] \bar{T}(s, P)$.

Matrix elements of this equation can be formed in the relative-momentum representation, using operator multiplication defined as

$$
\left\langle\overrightarrow{\mathrm{p}}|A B| \overrightarrow{\mathrm{p}}^{\prime}\right\rangle=(2 \pi)^{-3} \int d \overrightarrow{\mathrm{q}}\langle\overrightarrow{\mathrm{p}}|A| \overrightarrow{\mathrm{q}}\rangle\left\langle\overrightarrow{\mathrm{q}}|B| \overrightarrow{\mathrm{p}}^{\prime}\right\rangle .
$$

Comparison with Eqs. (6) and (8) then leads to the following identifications: 


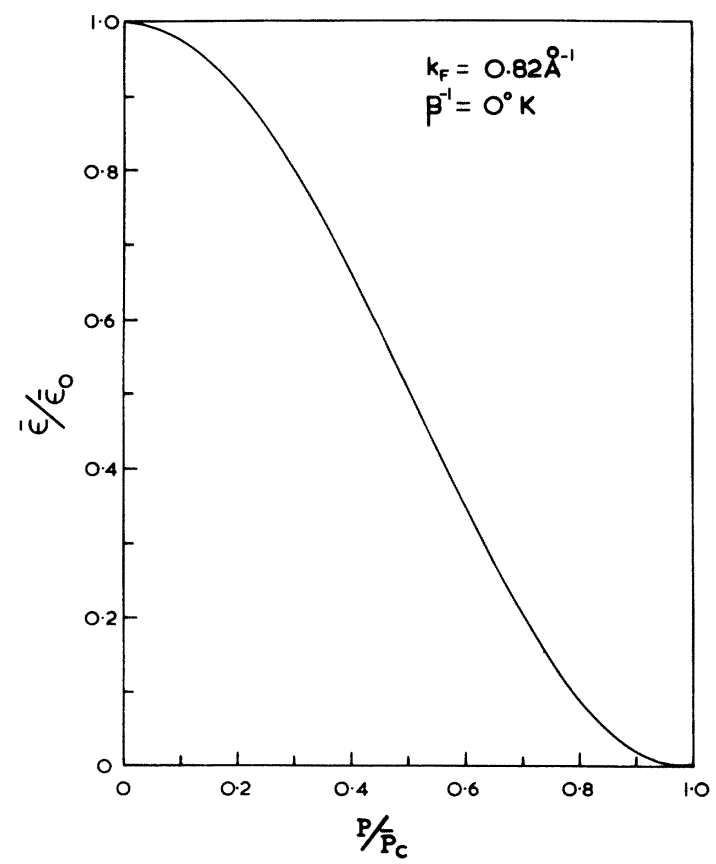

FIG. 2. Binding energy $\bar{\epsilon}$, as a function of the average pair momentum $P$, at zero temperature and a Fermi momentum $k_{F}=0.82 \AA^{-1}$, for the $S$-wave GF bound pair in liquid ${ }^{3} \mathrm{He}$, using the MFM potential (Refs. 18, 19), taken from Ref. 17. For these parameters, the curve can be fitted to Eq. (11) with $\bar{\epsilon}_{0}=-0.607 \AA^{-2}, \bar{P}_{c}=0.566 \AA^{-1}$, and $\bar{\lambda}=2.33$.

$$
\begin{aligned}
& \left\langle\overrightarrow{\mathrm{p}}|\bar{T}(s, P)| \overrightarrow{\mathrm{p}}^{\prime}\right\rangle=\bar{T}\left(\overrightarrow{\mathrm{p}}, \overrightarrow{\mathrm{p}}^{\prime} ; s, P\right), \\
& \left\langle\overrightarrow{\mathrm{p}}|u| \overrightarrow{\mathrm{p}}^{\prime}\right\rangle=u\left(\left|\overrightarrow{\mathrm{p}}-\overrightarrow{\mathrm{p}}^{\prime}\right|\right), \\
& \left\langle\overrightarrow{\mathrm{p}}|Q(P)| \overrightarrow{\mathrm{p}}^{\prime}\right\rangle=(2 \pi)^{3} \delta\left(\overrightarrow{\mathrm{p}}-\overrightarrow{\mathrm{p}}^{\prime}\right) Q(\overrightarrow{\mathrm{p}}, P), \\
& \left\langle\overrightarrow{\mathrm{p}}|\tilde{\mathrm{Q}}(P)| \overrightarrow{\mathrm{p}}^{\prime}\right\rangle=(2 \pi)^{3} \delta\left(\overrightarrow{\mathrm{p}}-\overrightarrow{\mathrm{p}}^{\prime}\right) \tilde{Q}(\overrightarrow{\mathrm{p}}, P), \\
& \left\langle\overrightarrow{\mathrm{p}}\left|g_{0}(s)\right| \overrightarrow{\mathrm{p}}^{\prime}\right\rangle=(2 \pi)^{3} \delta\left(\overrightarrow{\mathrm{p}}-\overrightarrow{\mathrm{p}}^{\prime}\right) g_{0}(\overrightarrow{\mathrm{p}}, s) .
\end{aligned}
$$

If Eq. (12) has a unique solution, then it is easy to show, using Eq. (4), that $\bar{T}$ must also be a solution of the alternative integral equation

$$
\bar{T}(s, P)=u-\bar{T}(s, P)\left[Q(P) g_{0}(s)-\bar{Q}(P) g_{0}^{\dagger}(s)\right] u .
$$

Equations (12) and (14) can be combined respectively with their own Hermitian conjugates to eliminate the potential $u$, thereby giving the relations

$$
\begin{aligned}
\bar{T}(s, P) & -\bar{T}^{\dagger}(s, P) \\
& =-\bar{T}^{\dagger}(s, P)[Q(P)+\tilde{Q}(P)]\left[g_{0}(s)-g_{0}^{\dagger}(s)\right] \bar{T}(s, P) \\
& =-\bar{T}(s, P)[Q(P)+\tilde{Q}(P)]\left[g_{0}(s)-g_{0}^{\dagger}(s)\right] \bar{T}^{\dagger}(s, P) .
\end{aligned}
$$

These equations express the important result that $\bar{T}$ satisfies a generalized unitarity relation. Taking matrix elements in the relative-momentum rep- resentation and decomposing into partial waves gives the equivalent forms

$$
\begin{aligned}
\bar{T}_{l}\left(p, p^{\prime} ; s, P\right) & =\bar{T}_{l}^{*}\left(p, p^{\prime} ; s, P\right), \quad s<0, \\
\bar{T}_{l}\left(p, p^{\prime} ; s, P\right) & -\bar{T}_{l}^{*}\left(p, p^{\prime} ; s, P\right) \\
= & (\kappa / 2 \pi i)[Q(\kappa, P)+\tilde{Q}(\kappa, P)] \bar{T}_{l}(p, \kappa ; s, P) \\
& \times \bar{T}_{l}^{*}\left(\kappa, p^{\prime} ; s, P\right), \quad s>0,
\end{aligned}
$$

where $\kappa \equiv+s^{1 / 2}$ for $s>0$. Setting $p=p^{\prime}=\kappa$ in Eq. (16) and defining $\bar{T}_{l}(\kappa, P) \equiv \bar{T}_{l}\left(\kappa, \kappa ; \kappa^{2}, P\right)$ to be the on-shell $T$ matrix, shows that this fully on-shell quantity is required to be unimodular, apart from some trivial real kinematic and phase-space factors. The on-shell $T$ matrix can thus be parametrized in terms of a real effective phase shift $\bar{\delta}_{l}(\kappa, P)$ as

$$
\begin{aligned}
\bar{T}_{l}(\kappa, P)= & -4 \pi \kappa^{-1}[Q(\kappa, P)+\tilde{Q}(\kappa, P)]^{-1} \\
& \times e^{i \bar{\delta}_{l}(\kappa, P)} \sin \bar{\delta}_{l}(\kappa, P) .
\end{aligned}
$$

These results can be summarized as follows. In a many-body medium having a temperature $\beta^{-1}$ and chemical potential $\mu$, the fully off-shell GF $T$ matrix is the solution of the nonrelativistic analog of the Bethe-Salpeter equation, ${ }^{20}$ given by our Eq. (12). It satisfies a (modified) two-particle unitarity relation, Eq. (15), which in turn leads to the parametrization of elastic scattering of two particles or two holes in the medium by effective phase shifts, Eq. (17). The development of these equations closely parallels the development of two-particle potential scattering in free space. ${ }^{21}$ Under the replacement $Q \rightarrow 1, \tilde{Q} \rightarrow 0$, Eqs. (12) $-(17)$ give a complete description of two-body scattering by a potential $u$. In particular, Eq. (14) reduces to the ordinary two-particle Lippmann-Schwinger equation. It should be emphasized that Eq. (12) contains implicit boundary conditions which require the scattering solutions to behave asymptotically (in the large separation limit) as linear combinations of outgoing waves (for two particles) and incoming waves (for two holes). This has the consequence that the $T$ matrix is analytic in neither the upper nor the lower half of the complex $s$ plane. It is useful to define an alternate form of the GF $T$ matrix, namely $\bar{T}^{(+)}(s, P)$, which is analytic in the entire positive imaginary (upper half) $s$ plane, and for which the scattering solutions behave asymptotically as pure outgoing waves:

$$
\begin{aligned}
\bar{T}^{(+)}(s, P) & =u-u[Q(P)-\tilde{Q}(P)] g_{0}(s) \bar{T}^{(+)}(s, P) \\
& =u-\bar{T}^{(+)}(s, P)[Q(P)-\tilde{Q}(P)] g_{0}(s) u .
\end{aligned}
$$

The $T$ matrices $\bar{T}(s, P)$ and $\bar{T}^{(+)}(s, P)$ have similar properties, and can be simply related by the equa- 
tion

$$
\begin{aligned}
\bar{T}(s, P) & -\bar{T}^{(+)}(s, P) \\
= & -\bar{T}^{(+)}(s, P) \tilde{Q}(P)\left[g_{0}(s)-g_{0}^{+}(s)\right] \bar{T}(s, P),
\end{aligned}
$$

which can be written equivalently in a partialwave representation as,

$$
\begin{aligned}
\bar{T}_{l}\left(p, p^{\prime} ; s, P\right)= & \bar{T}_{l}^{(+)}\left(p, p^{\prime} ; s, P\right), \quad s<0 \\
\bar{T}_{l}\left(p, p^{\prime} ; s, P\right)- & \bar{T}_{l}^{(+)}\left(p, p^{\prime} ; s, P\right) \\
= & (\kappa / 2 \pi i) \tilde{Q}(\kappa, P) \bar{T}_{l}^{(+)}(p, \kappa ; s, P) \\
& \times \bar{T}_{l}\left(\kappa, p^{\prime} ; s, P\right), \quad s>0
\end{aligned}
$$

and where $\kappa \equiv+s^{1 / 2}$ as before, for $s>0$. Exactly as in Eq. (17), one can define phase shifts $\bar{\delta}_{l}^{(+)}(\kappa, P)$ by taking the on-shell version of Eq. (20). One then finds

$$
\begin{aligned}
\bar{T}_{l}^{(+)}(\kappa, P) \equiv & \bar{T}_{l}^{(+)}\left(\kappa, \kappa ; \kappa^{2}, P\right) \\
= & -4 \pi \kappa^{-1}[Q(\kappa, P)-\tilde{Q}(\kappa, P)] \\
& \times e^{i \bar{\delta}_{l}^{(+)}(\kappa, P)} \sin \bar{\delta}_{l}^{(+)}(\kappa, P),
\end{aligned}
$$

where the relation between the two phase shifts $\bar{\delta}_{l}$ and $\bar{\delta}_{l}^{(+)}$is

$$
\cot \bar{\delta}_{l}^{(+)}(\kappa, P)=\left(\frac{Q(\kappa, P)+\tilde{Q}(\kappa, P)}{Q(\kappa, P)-\bar{Q}(\kappa, P)}\right) \cot \bar{\delta}_{l}(\kappa, P) .
$$

\section{WAVE EQUATIONS AND A GENERALIZED LEVINSON'S THEOREM}

Although the GF $T$ matrix is the essential quantity which serves to calculate the proper self energy function, the modified form defined by Eq. (18) leads to a description of pair scattering in the many-body medium which closely parallels the usual picture of free two-particle scattering. In this picutre we have particles described by asymptotically free states, interacting via a twobody potential, and giving rise to well-defined scattered waves outside the range of the interaction. Using the two-particle Green's function $\bar{g}(s, P)$ and a generalized wave operator $\bar{X}(s, P)$, defined operationally as

$$
u \bar{g}(s, P) \equiv \bar{T}^{(+)}(s, P) g_{0}(s), u \bar{X}(s, P) \equiv \bar{T}^{(+)}(s, P),
$$

then Eq. (18) can be represented as,

$$
\begin{aligned}
\bar{g}^{-1}(s, P) & =g_{0}^{-1}(s)+[Q(P)-\tilde{Q}(P)] u \\
& \equiv \bar{L}(P)-s-i \eta,
\end{aligned}
$$

where it is immediately seen from Eq. (6) that $\bar{E}(P)$ is given by,

$$
\overline{\mathfrak{L}}(P)=\frac{1}{2} H_{0}+[Q(P)-\tilde{Q}(P)] u \text {. }
$$

The operator $\bar{d}(P)$ is non-Hermitian, and we shall initially assume that its eigenvalue spectrum $\bar{s}_{\nu}(P)$ is composed of two branches, viz. a continuous positive energy branch $\left[\nu \rightarrow \overrightarrow{\mathrm{k}} ; \bar{s}_{\nu}(P) \rightarrow \overrightarrow{\mathrm{k}}^{2}\right]$, and a discrete branch having $N_{l}$ bound states in the $l$ th relative partial-wave channel $\left[\nu \rightarrow(i, l) ; i=1, \cdots, N_{l} ; \bar{s}_{\nu}(P)-\bar{s}_{i l}(\boldsymbol{P})<0\right]$. We also assume that the corresponding eigenvectors form a basis for $\overline{\mathscr{L}}(P)$,

$$
\begin{aligned}
& {\left[\overline{\mathfrak{L}}(P)-\bar{s}_{\nu}(P)\right]\left|\bar{\phi}_{\nu}(P)\right\rangle=0,} \\
& \bar{s}_{\nu}(P)=\left\{\begin{array}{l}
\overrightarrow{\mathrm{k}}^{2}, \quad \nu=\overrightarrow{\mathrm{k}} \\
\bar{s}_{i l}(P), \quad \nu=(i, l) ; i=1, \cdots, N_{l} .
\end{array}\right.
\end{aligned}
$$

With these assumptions it necessarily follows that there exists a reciprocal basis ${ }^{22}\left|\bar{\psi}_{\nu}(P)\right\rangle$, satisfying the equation,

$$
\left[\overline{\mathfrak{L}}^{\dagger}(P)-\bar{s}_{\nu}^{*}(P)\right]\left|\bar{\psi}_{\nu}(P)\right\rangle=0,
$$

and in terms of which the usual completeness and orthonormality conditions are

$$
\sum_{\nu}\left|\bar{\phi}_{\nu}(P)\right\rangle\left\langle\bar{\psi}_{\nu}(P)\right|=1,\left\langle\bar{\psi}_{\nu}(P) \mid \bar{\phi}_{\nu^{\prime}}(P)\right\rangle=\delta_{\nu \nu^{\prime}} .
$$

We shall focus attention on the positive energy solutions of Eq. (25) having outgoing-wave boundary conditions at infinity. In a relative-momentum representation,

$$
\bar{\phi}_{\overrightarrow{\mathrm{k}}}(\overrightarrow{\mathrm{p}} ; P) \equiv\left\langle\overrightarrow{\mathrm{p}} \mid \bar{\phi}_{\overrightarrow{\mathrm{k}}}(P)\right\rangle,
$$

we find by comparison with Eqs. (23) and (24),

$$
\bar{\phi}_{\overrightarrow{\mathbf{k}}}(\overrightarrow{\mathrm{p}} ; P)=\left\langle\overrightarrow{\mathrm{p}}\left|\bar{X}\left(k^{2}, P\right)\right| \overrightarrow{\mathrm{k}}\right\rangle
$$

and

$$
\begin{aligned}
\bar{\phi}_{\overrightarrow{\mathrm{k}}}(\overrightarrow{\mathrm{p}} ; P)= & (2 \pi)^{3} \delta(\overrightarrow{\mathrm{p}}-\overrightarrow{\mathrm{k}}) \\
& -\frac{Q(\overrightarrow{\mathrm{p}}, P)-\tilde{Q}(\overrightarrow{\mathrm{p}}, P)}{p^{2}-k^{2}-i \eta} \bar{T}^{(+)}\left(\overrightarrow{\mathrm{p}}, \overrightarrow{\mathrm{k}} ; k^{2}, P\right) .
\end{aligned}
$$

The pair wave function in coordinate space is accordingly given in terms of the half-shell $T$ matrix $\bar{T}^{(+)}$by

$$
\begin{aligned}
\bar{\phi}_{\overrightarrow{\mathrm{k}}}(\overrightarrow{\mathrm{r}} ; P)=e^{i \overrightarrow{\mathrm{k}} \cdot \overrightarrow{\mathrm{r}}}- & \int \frac{d \overrightarrow{\mathrm{p}}}{(2 \pi)^{3}} e^{\mathrm{i} \overrightarrow{\mathrm{p}} \cdot \overrightarrow{\mathrm{r}}}\left(\frac{Q(\overrightarrow{\mathrm{p}}, P)-\tilde{Q}(\overrightarrow{\mathrm{p}}, P)}{p^{2}-k^{2}-i \eta}\right) \\
& \times \bar{T}^{(+)}\left(\overrightarrow{\mathrm{p}}, \overrightarrow{\mathrm{k}} ; k^{2}, P\right) .
\end{aligned}
$$

The asymptotic form of this wave function, $\bar{\phi}_{\overrightarrow{\mathrm{k}}}(\overrightarrow{\mathrm{r}} ; P)$, in the limit $r \rightarrow \infty$, can easily be found in the following way. The $T$ matrix is defined in a mixed representation, by

$$
\bar{T}^{(+)}\left(\overrightarrow{\mathrm{r}}, \overrightarrow{\mathrm{k}} ; k^{2}, P\right)=(2 \pi)^{-3} \int d \overrightarrow{\mathrm{p}} e^{i \overrightarrow{\mathrm{p}} \cdot \overrightarrow{\mathrm{r}}} \bar{T}^{(+)}\left(\overrightarrow{\mathrm{p}}, \overrightarrow{\mathrm{k}} ; k^{2}, P\right),
$$

so that the pair wave function can be written in the form 


$$
\bar{\phi}_{\overrightarrow{\mathrm{k}}}(\overrightarrow{\mathrm{r}} ; P)=e^{i \overrightarrow{\mathrm{k}} \cdot \overrightarrow{\mathrm{r}}}-\int d \overrightarrow{\mathrm{r}}^{\prime}\left[\int \frac{d \overrightarrow{\mathrm{p}}}{(2 \pi)^{3}} e^{i \overrightarrow{\mathrm{p}} \cdot\left(\overrightarrow{\mathrm{r}}-\overrightarrow{\mathrm{r}}^{\prime}\right)}\left(\frac{Q(\overrightarrow{\mathrm{p}}, P)-\tilde{Q}(\overrightarrow{\mathrm{p}}, P)}{p^{2}-k^{2}-i \eta}\right)\right] \bar{T}^{(+)}\left(\overrightarrow{\mathrm{r}}, \overrightarrow{\mathrm{k}} ; k^{2}, P\right) .
$$

In the limit $|\vec{r}| \rightarrow \infty$, the term in brackets becomes

$$
\int \frac{d \overrightarrow{\mathrm{p}}}{(2 \pi)^{3}} e^{i \overrightarrow{\mathrm{p}} \cdot \overrightarrow{\mathrm{R}}}\left(\frac{Q(\overrightarrow{\mathrm{p}}, P)-\tilde{Q}(\overrightarrow{\mathrm{p}}, P)}{p^{2}-k^{2}-i \eta}\right) \underset{R \rightarrow \infty}{\longrightarrow} \frac{e^{i k R}}{4 \pi R}[Q(k, P)-\tilde{Q}(k, P)] 。
$$

Substituting back into the previous expression, and making use of the expansion,

$\frac{e^{i k\left|\overrightarrow{\mathrm{r}}-\overrightarrow{\mathrm{r}}^{\prime}\right|}}{k\left|\overrightarrow{\mathrm{r}}-\overrightarrow{\mathrm{r}}^{\prime}\right|}=\sum_{l=0}^{\infty}(2 l+1) j_{l}\left(k r^{\prime}\right) h_{l}^{(+)}(k r) P_{l}\left(\hat{\gamma} \circ \hat{r}^{\prime}\right), \quad r>r^{\prime}$

gives the desired results,

$\bar{\phi}_{\overrightarrow{\mathrm{k}}}(\overrightarrow{\mathrm{r}} ; P) \underset{r \rightarrow \infty}{\longrightarrow} e^{i \overrightarrow{\mathrm{k}} \cdot \overrightarrow{\mathrm{r}}}-\left(e^{i k r} / r\right) \bar{f}(\overrightarrow{\mathrm{k}} \cdot \hat{r} ; P)$,

$\bar{f}(\overrightarrow{\mathrm{k}} \cdot \hat{r} ; P)=(4 \pi)^{-1}[Q(k, P)-\tilde{Q}(k, P)]$

$$
\begin{gathered}
\times \sum_{i=0}^{\infty}(2 l+1) P_{l}(\hat{\gamma} \cdot \hat{k}) \bar{T}_{l}^{(+)}\left(k, k ; k^{2}, P\right) \\
=(2 i k)^{-1} \sum_{l=0}^{\infty}(2 l+1) P_{l}(\hat{k} \cdot \hat{\gamma}) \\
\times\left[e^{2 i \bar{\sigma}_{l}^{(+)}(k ; P)}-1\right],
\end{gathered}
$$

and

$\bar{\phi}_{k l}(r ; P) \underset{r \rightarrow \infty}{\longrightarrow} \frac{i^{l}}{k r} e^{i \bar{\delta}_{l}^{(+)}(k ; P)} \sin \left[k r-\frac{1}{2} l \pi+\bar{\delta}_{l}^{(+)}(k ; P)\right]$,

where

$$
\bar{\phi}_{\overrightarrow{\mathbf{k}}}(\overrightarrow{\mathrm{r}} ; P)=\sum_{l=0}^{\infty}(2 l+1) P_{l}(\hat{k} \cdot \hat{r}) \bar{\phi}_{k l}(r ; P) .
$$

In a completely analogous manner, it is easy to show that

$$
\lim _{r \rightarrow \infty} \bar{\psi}_{\overrightarrow{\mathbf{k}}}(\overrightarrow{\mathrm{r}} ; P)=\lim _{r \rightarrow \infty} \bar{\phi}_{\overrightarrow{\mathrm{k}}}(\overrightarrow{\mathrm{r}} ; P),
$$

where

$$
\bar{\psi}_{\overrightarrow{\mathbf{k}}}(\overrightarrow{\mathrm{r}} ; P) \equiv\left\langle\overrightarrow{\mathbf{r}} \mid \bar{\psi}_{\overrightarrow{\mathbf{k}}}(P)\right\rangle \text {. }
$$

We shall demonstrate that, just as in free twobody scattering, the existence of bound states is directly related to the asymptotic behavior of the phase shifts in the same relative partial-wave channel. Using Eqs. (22), (25), (27), (30), and (31), an analogous relationship can be established between the asymptotic values of the GF phase shifts $\bar{\delta}_{l}$ and the number of bound pairs $N_{l}$ in a particular channel. We shall follow a procedure ${ }^{23}$ in which the size of the system is maintained as a free parameter until the end of the calculation.
When employed in our formalism, this process requires cancellations which arise from the interchange of different limits in the normalization of wave functions, as we shall see below. In what follows we shall adopt the policy that any ambiguities due to this procedure can be correctly resolved by first reformulating the scattering problem in a finite volume $\left(\sim a^{3}\right)$, then carrying out the prescribed operation, and finally proceeding to the infinite volume $(a \rightarrow \infty)$ limit. Again, we shall assume a many-body system containing $N_{l}$ bound pairs in the $l$ th partial-wave GF channel, together with a continuous branch of the two-body spectrum, as described by Eq. (25). We also consider that this spectrum of states satisfies the completeness relation of Eq. (27), which we now write as

$$
\sum_{\nu} \bar{\phi}_{\nu}(\overrightarrow{\mathrm{r}} ; P) \bar{\psi}_{\nu}^{*}\left(\overrightarrow{\mathrm{r}}^{\prime} ; P\right)=(2 \pi)^{-3} \int d \overrightarrow{\mathrm{k}} e^{i \overrightarrow{\mathrm{k}} \cdot\left(\vec{r}-\vec{r}^{\prime}\right)},
$$

or by decomposing into partial waves as

$$
\begin{aligned}
& \sum_{i=1}^{N_{l}} \bar{\phi}_{i l}(r ; P) \bar{\psi}_{i l} *\left(r^{\prime} ; P\right) \\
& \quad=\int_{0}^{\infty} \frac{d k k^{2}}{2 \pi^{2}}\left[j_{l}(k r) j_{l}\left(k r^{\prime}\right)-\bar{\phi}_{k l}(r ; P) \bar{\psi}_{k l}^{*}\left(r^{\prime} ; P\right)\right] .
\end{aligned}
$$

By setting $r^{\prime}=r$ in the above equation, integrating the resulting equation over all space, and using the orthonormality relation of Eq. (27), we obtain the basic relation,

$$
\frac{N_{l}}{4 \pi}=\lim _{a \rightarrow \infty} \int_{0}^{\infty} \frac{d k k^{2}}{2 \pi^{2}}\left(\int_{0}^{a} d r r^{2} j_{l}^{2}(k r)-I_{k l}(a)\right),
$$

where

$$
I_{k l}(a) \equiv \int_{0}^{a} d r r^{2} \bar{\phi}_{k l}(r ; P) \bar{\psi}_{k l}^{*}(r ; P)
$$

The calculation of $I_{k l}(a)$ now follows from the coordinate-space representation of Eqs. (25) and (26) in a particular relative partial wave $l$, 


$$
\begin{aligned}
& \left(D_{r}^{2}-\frac{l(l+1)}{r^{2}}+k^{2}\right) \bar{\phi}_{k l}(r ; P) \\
& -4 \pi \int_{0}^{\infty} d r^{\prime} r^{\prime 2} F_{l}\left(r, r^{\prime} ; P\right) u\left(r^{\prime}\right) \bar{\phi}_{k l}\left(r^{\prime} ; P\right)=0, \\
& \left(D_{r}^{2}-\frac{l(l+1)}{r^{2}}+k^{2}\right) \bar{\psi}_{k l}(r ; P) \\
& -4 \pi u(r) \int_{0}^{\infty} d r^{\prime} r^{\prime 2} F_{l}\left(r, r^{\prime} ; P\right) \bar{\psi}_{k l}\left(r^{\prime} ; P\right)=0,
\end{aligned}
$$

where

$$
D_{r}^{2}=\frac{1}{r^{2}} \frac{d}{d r}\left(r^{2} \frac{d}{d r}\right)
$$

and

$$
\begin{aligned}
F_{l}\left(r, r^{\prime} ; P\right)=\int_{0}^{\infty} \frac{d q q^{2}}{2 \pi^{2}} j_{l}(q r)[Q(q, P) & \\
& -\tilde{Q}(q, P)] j_{l}\left(q r^{\prime}\right) .
\end{aligned}
$$

The first of Eqs. (33) is now differentiated with respect to $k$ (where we denote such differentiation by a prime), after which it is multiplied by the factor $r^{2} \bar{\psi}_{k l}^{*}(r ; P)$; and the complex conjugate of the second of Eqs. (33) is multiplied by the factor $r^{2} \bar{\phi}_{k l}^{\prime}(r ; P)$. The difference of the resulting two equations is then taken, and after integrating on the variable $r$ over the range $(0, a)$, we obtain the result

$$
\begin{aligned}
I_{k l}(a)=(-2 k)^{-1} \int_{0}^{a} d r r^{2} & {\left[\bar{\psi}_{k l}^{*}(r ; P) D_{r}^{2} \bar{\phi}_{k l}^{\prime}(r ; P)\right.} \\
& \left.-\bar{\phi}_{k l}^{\prime}(r ; P) D_{r}^{2} \bar{\psi}_{k l}^{*}(r ; P)\right],
\end{aligned}
$$

where terms which vanish as $a$ tends to infinity have been ignored. Integrating this final result by parts, and using the asymptotic form of the wave functions given by Eqs. (30) and (31), one obtains

$$
\begin{aligned}
I_{k l}(a)= & \frac{a}{2 k^{2}}+\frac{1}{2 k^{2}} \bar{\delta}_{l}^{(+)^{\prime}}(k ; P) \\
& -\frac{1}{4 k^{3}} \sin \left[2 k a-l \pi+2 \bar{\delta}_{l}^{(+)}(k ; P)\right],
\end{aligned}
$$

as a correct result in the limit as $a \rightarrow \infty$. This equation, together with the relation

$$
\int_{0}^{a} d r r^{2} j_{l}^{2}(k r) \underset{a \rightarrow \infty}{\rightarrow} \frac{a}{2 k^{2}}-\frac{\sin (2 k a-l \pi)}{4 k^{3}},
$$

when substituted into the basic relation of Eq. (32), gives

$$
\begin{aligned}
\pi N_{l}=\bar{\delta}_{l}^{(+)}(0 ; P)-\bar{\delta}_{l}^{(+)}(\infty ; P) & \\
+\frac{1}{2} \int_{0}^{\infty} \frac{d z}{z} & \left\{\sin \left[z-l \pi+2 \bar{\delta}_{l}^{(+)}(0 ; P)\right]\right. \\
& -\sin (z-l \pi)\}
\end{aligned}
$$

where we have made the substitution $z=2 k a$, and have taken the limit $a \rightarrow \infty$. This equation has solutions for the asymptotic phase shifts, given by

$$
\left(N_{l}+\lambda\right) \pi=\bar{\delta}_{l}^{(+)}(0 ; P)-\bar{\delta}_{l}^{(+)}(\infty ; P),
$$

where

$$
\lambda=\left\{\begin{array}{l}
0, \quad \bar{\delta}_{l}^{(+)}(0 ; P)=n \pi \\
\frac{1}{2}(-1)^{l}, \quad \bar{\delta}_{i}^{(+)}(0 ; P)=\left(n+\frac{1}{2}\right) \pi,
\end{array}\right.
$$

where $n=1,2, \ldots, \infty$. If the scattering amplitude does not have any poles at exactly zero energy, then $\lambda$ is always zero. That is, apart from the rather artificial case of a bound state of exactly zero energy, we have the result

$$
\pm \bar{\delta}_{l}(0 ; P)+\bar{\delta}_{l}(\infty ; P)=-N_{l} \pi, \quad P^{2} \lessgtr \mu
$$

where we have used Eq. (22) to relate the two phase shifts $\bar{\delta}_{l}$ and $\bar{\delta}_{l}^{(+)}$. This result is the exact

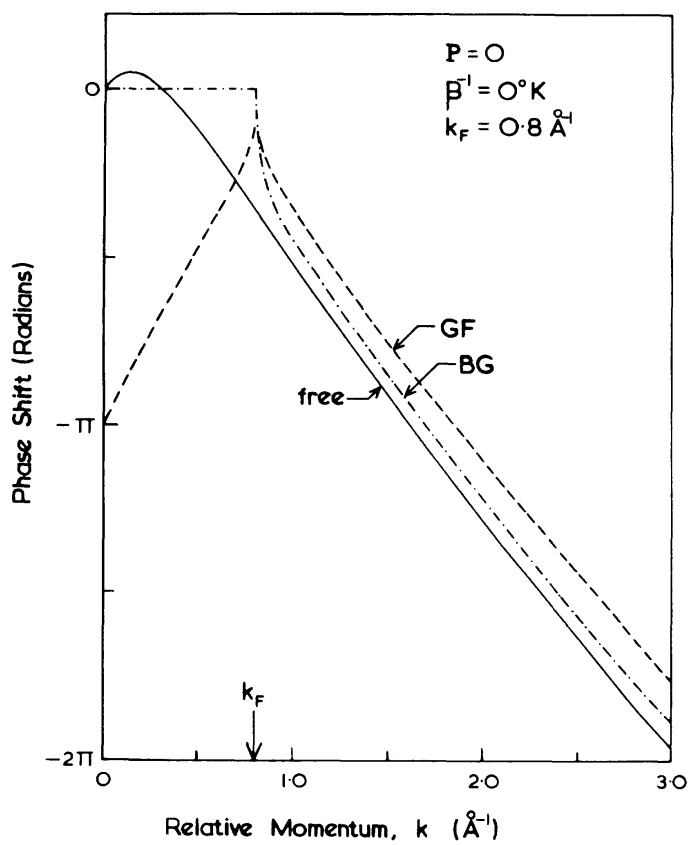

FIG. 3. Comparison of the $S$-wave free scattering phase shift with the corresponding effective phase shifts in the GF and BG formalisms. The GF effective phase shift is defined in Eq. (17), and the corresponding BG quantity is obtained similarly by turning off hole-hole scattering $(\tilde{Q} \rightarrow 0)$ throughout. The potential is the MFM (Refs. 18, 19) appropriate to liquid ${ }^{3} \mathrm{He}$, and the curves are shown at zero temperature and a Fermi momentum, $k_{F} \doteq 0.8 \AA^{-1}$, and for zero total momentum of the pair. The presence of a single GF bound state, and the absence of either a free or a BG bound state can be seen by using the appropriate (modified) form of Levinson's theorem [ Eq. (34)] in each case. (Note that each of the phase shifts tends to zero at infinite relative momentum $k$.) 
GF analog for pair scattering in the many-body medium of the usual Levinson's theorem for free scattering, and in particular the sign of the righthand side of Eq. (34) should be noted. A numerical example illustrating the result of Eq. (34), as calculated in Ref. 17, is shown in Fig. 3.

\section{SUMMARY AND CONCLUSIONS}

In summary we wish to emphasize the following points. The simultaneous description through the GF formalism of both pairs of particles and pairs of holes embedded in a many-body medium of identical particles, can be placed in a close correspondence with two-particle scattering in free space. The fully off-shell scattering amplitude in this formalism is the solution of the nonrelativistic analog of the Bethe-Salpeter equation. It satisfies a generalized unitarity condition which is the analog of two-particle unitarity, and it similarly leads to a parametrization of the positiveenergy on-shell GF $T$ matrix (corresponding to scattering solutions) in terms of real effective phase shifts. Also, nonlocal wave equations for the scattering states can be obtained with appropriate boundary conditions, and the asymptotic form of the pair wave functions can be related to the on-shell GF $T$ matrix in a way which is form equivalent to the free scattering counterpart. The dependence of this asymptotic form on the temperature and chemical potential of the system is solely restricted to the dependence of the effective phase shifts on these quantities.

As in free scattering, the GF $T$ matrix can have bound-state poles, the existence of which will, in general, depend on the properties of the kernel of the $T$-matrix equation (or the equivalent wave equations). This kernel depends parametrically on the thermodynamic properties of the medium, and also on the total linear momentum of the interacting pair. The properties of this kernel are different from those of both the corresponding free scattering equation and the BG equation which neglects simultaneous hole-hole scattering. In particular, for a potential for which there exist no bound states in the free two-body system, the GF matter $T$ matrix can sustain bound states which depend implicitly on the properties of the medium (and see Ref. 9). The existence of bound pairs in the medium (being linear combinations of particleparticle and hole-hole states) having a negative total energy can be inferred from the behavior of the positive-energy on-shell scattering amplitude via a modified form of Levinson's theorem.

For a many-body system in which the GF $T$ matrix contains a (bound-state) pole, the expansion for the proper self-energy contains additional terms which can be written in the form

$$
\bar{\Sigma} *\left(\overrightarrow{\mathrm{p}}, p_{0}\right)=\bar{\Sigma}_{\text {pole }}^{*}+\bar{\Sigma}_{\text {background }}^{*},
$$

where the contribution from the so-called background term is exactly that given by the usual expressions for $\bar{\Sigma}^{*}$ in the absence of any poles. Because of the pole, there will be combinations of particular values of $\overrightarrow{\mathrm{p}}$ and $p_{0}$ for which $\bar{\Sigma}_{\text {pole }}^{*}$ and $\bar{\Sigma}_{\text {background }}^{*}$ will become infinite, which, by Eq. (3), will make an important contribution to the singleparticle energy spectrum and to the thermodynamic functions describing the system. Suffice it to say that numerical calculations for these systems are difficult. We are currently performing such computations for (the normal phase of) liquid ${ }^{3} \mathrm{He}$ and for nucleonic matter, and shall report them elsewhere.
*Permanent address.

${ }^{1}$ K. A. Brueckner, C. A. Levinson, and H. M. Mahmoud, Phys. Rev. 95, 217 (1954).

${ }^{2}$ L. D. Landau,Zh. Eksp. Teor. Fiz. 30, 1058 (1956) [Sov. Phys.-JETP $\underline{3}, 920$ (1956)] ; $\underline{32}, 59$ (1957) [ㅁ, 101 (1957)].

${ }^{3} \mathrm{~J}$. Goldstone, Proc. R. Soc. Lond. A 239, 267 (1957).

${ }^{4}$ L. N. Cooper, Phys. Rev. 104, 1189 (1956).

${ }^{5}$ C. N. Yang, Revs. Mod. Phys. 34, 694 (1962).

${ }^{6} \mathrm{~W}$. Kohn and D. Sherrington, Revs. Mod. Phys. 42,1 (1970).

${ }^{7}$ R. P. Feynman, Statistical Mechanics: A Set of Lectures (Benjamin, Reading, Mass., 1972).

${ }^{8}$ K. A. Brueckner and J. L. Gammel, Phys. Rev. 109 , 1040 (1958); K. A. Brueckner, T. Soda, P. W. Anderson, and P. Morel, Phys. Rev. 118, 43 (1960).

${ }^{9}$ R. F. Bishop, M. R. Strayer and J. M. Irvine, Phys. Rev. A 10, 2423 (1974); J. Low Temp. Phys. 20, 571
(1975).

${ }^{10}$ V. M. Galitskii and A. B. Migdal, Zh. Eksp. Teor. Fiz. 34, 139 (1958) [Sov. Phys.-JETP 7, 96 (1958)].

${ }^{11}$ A. A. Abrikosov, L. P. Gorkov, and I. E. Dzyaloshin skii, Zh. Eksp. Teor. Fiz. 36, 900 (1959) [Sov. Phys.JETP 9, 636 (1959)]; J. M. Luttinger and J. C. Ward, Phys. Rev. 118, 1417 (1960).

${ }^{12}$ R. F. Bishop, Ann. Phys. (N.Y.) 77, 106 (1973).

${ }^{13}$ Recent Bethe-Goldstone calculations for liquid ${ }^{3} \mathrm{He}$ and nuclear matter are cited in H. B. Ghassib, R. H. Ibarra and J. M. Irvine, Ann. Phys. (N.Y.) 85, 378 (1973); and J. P. Jeukenne, A. Lejeune, and C. Mahaux, Nucl. Phys. A 245, 411 (1975), respectively.

${ }^{14} \mathrm{H}$. A. Bethe and J. Goldstone, Proc. R. Soc. Lond. A $\underline{238}, 551$ (1957).

${ }^{15}$ A. L. Fetter and J. D. Walecka, Quantum Theory of Many-Particle Systems (McGraw-Hill, New York, 1971). 
${ }^{16} \mathrm{We}$ assume here, and henceforth, a static, spin-independent, uniform infinite system.

${ }^{17}$ H. B. Ghassib, R. F. Bishop, and M. R. Strayer, J. Low Temp. Phys. (to be published).

${ }^{18}$ A. A. Frost and B. Musulin, J. Chem. Phys. 22, 1017 (1954).

${ }^{19}$ R. F. Bishop, H. B. Ghassib and M. R. Strayer, J. Chem. Phys. (to be published).
${ }^{20}$ E. E. Salpeter and H. A. Bethe, Phys. Rev. $\underline{84}, 1232$ (1951).

${ }^{21} \mathrm{~V}$. de Alfaro and T. Regge, Potential Scattering (NorthHolland, Amsterdam, 1965).

${ }^{22}$ B. Friedman, Principles and Techniques of Applied Mathematics, (Wiley, New York, 1956).

${ }^{23}$ This technique is based in part on unpublished lecture notes by F. Villars, M.I.T., 1969. 